\title{
Further evidence that Norway rats do not socially transmit learned aversions to toxic baits
}

\author{
BENNETT G. GALEF, JR., LAUREL M. MCQUOID, and ELAINE E. WHISKIN \\ McMaster University, Hamilton, Ontario, Canada
}

\begin{abstract}
Three experiments were undertaken to examine the effects of interactions with demonstrator rats made ill by injection of lithium chloride $(\mathrm{LiCl})$ on the later food choices of their observers. We found that (1) observer rats that had been taught an aversion to an unfamiliar diet exhibited a substantial reduction of that aversion after interacting with poisoned demonstrators that had eaten the diet to which the observers had learned an aversion, (2) exposure of an observer rat to poisoned demonstrator rats that had eaten a diet interfered with later acquisition by the observer of an aversion to the diet that the poisoned demonstrators had eaten, and (3) after interacting with poisoned demonstrators that had eaten one of two diets, observers that ate both diets and were then made ill formed an aversion to whichever diet their respective, poisoned demonstrators had not eaten. The present experiments, like previous studies both in our laboratory and elsewhere, failed to provide any evidence that naive observer rats will learn to avoid a food as a result of interacting with demonstrator rats that had eaten the food and exhibit symptoms of toxicosis. To the contrary, observer rats in the present experiments exhibited an enhanced preference for foods eaten by sick demonstrators.
\end{abstract}

After naive rats or mice (observers) interact with recently fed conspecifics (demonstrators), the observers exhibit enhancement of their preferences for the foods that their respective demonstrators had eaten (Galef \& Wigmore, 1983; Posadas-Andrews \& Roper, 1983; Richard, Grover, \& Davis, 1987; Valsecchi \& Galef, in press). The finding that rats can determine which foods conspecifics have recently eaten has led a number of investigators to inquire as to whether naive individuals will avoid foods eaten by obviously ill conspecifics. The results of such experiments, employing the straightforward procedure of introducing naive rats to demonstrators recently fed a novel food and then made ill by toxic injection, have been unequivocal: observer rats not only fail to avoid unfamiliar foods eaten by ill demonstrators, they exhibit increased preferences for foods eaten by ill demonstrators that are similar in magnitude to the increased preferences observer rats exhibit for foods eaten by healthy demonstrators (Galef \& Wigmore, 1983; Galef, Wigmore, \& Kennett, 1983; Grover et al., 1988; A. Posadas-Andrews, personal communication, 1982).

In the years since our laboratory first reported a failure to find social transmission of flavor aversions from demonstrator rats to their observers (Galef et al., 1983), we have developed a number of new procedures for examining social influences on food preference in rats. In particular, we have found that (1) many observers, taught

The research reported here was supported by grants from the Natural Sciences and Engineering Research Council of Canada and the McMaster University Research Board to B.G.G. We thank Mertice Clark for her thoughtful consideration of earlier drafts. Requests for reprints should be addressed to Bennett G. Galef, Jr., Department of Psychology, McMaster University, Hamilton, Ontario L8S 4K1, Canada. an aversion to an unfamiliar diet, exhibit substantial attenuation of that aversion following interaction with demonstrators that have just eaten the same diet (Galef, 1985b, 1986a); (2) preexposure of a naive observer rat to demonstrators that have eaten an unfamiliar diet attenuates or blocks subsequent learning by the observer of an aversion to the diet eaten by its respective demonstrators (Galef, 1989; Heyes \& Durlach, 1990); and (3) after interacting with demonstrators that have eaten one of two novel diets, observer rats that eat both diets and then are made ill form an aversion to whichever diet their respective demonstrators did not eat (Galef, 1986b, 1987).

The studies cited above used only nonpoisoned demonstrators. In the series of experiments presented below, we used the three procedures described above to examine the effects of interaction with demonstrators that had eaten an unfamiliar food and had then been injected with a lithium chloride ( $\mathrm{LiCl}$ ) solution on ingestion by observers of the diet eaten by their respective, poisoned demonstrators.

\section{EXPERIMENT 1}

In Experiment 1, we sought to determine whether naive observer rats that had formed an aversion to an unfamiliar, palatable diet would show attenuation of that aversion after interacting with sick demonstrators that had eaten that diet.

\section{Method}

\section{Subjects}

Forty-two experimentally naive 42-day-old female Long-Evans rats, born in the McMaster vivarium to breeding stock descended from animals purchased from Charles River Canada (St. Constant, Quebec), served as observers. An additional 48 49- to 56-day-old females obtained from the same source served as demonstrators. 


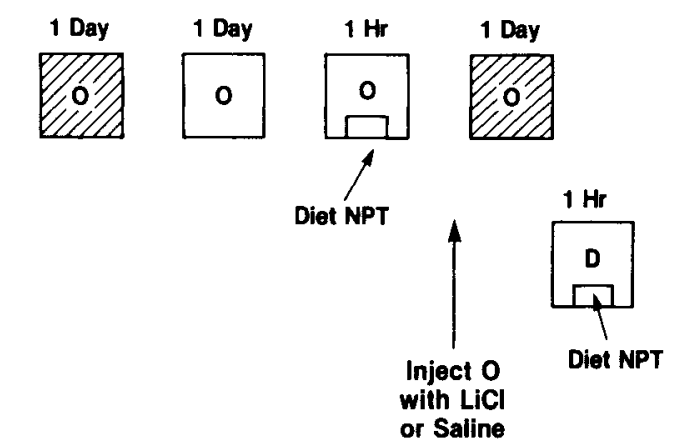

Step: 1
2
3
4

5

6

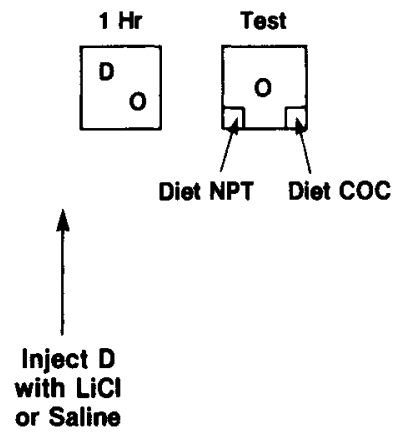

or Saline

Figure 1. Schematic of the procedure of Experiment 1. $D=$ demonstrator, $O=$ observer, hatching $=$ ad-lib access to Purina Rodent Lab Chow pellets.

\section{Apparatus}

The observers and demonstrators were housed in separate rooms in individual wire-mesh hanging cages $(22 \times 24 \times 27.5 \mathrm{~cm})$.

\section{Procedure}

Treatment of the observers and demonstrators during the experiment was as follows (see Figure 1).

Step 1 . The observers were placed individually in wire-mesh hanging cages and maintained for $24 \mathrm{~h}$ on ad-lib water and Purina Rodent Lab Chow pellets.

Step 2. Each observer was then food-deprived for $24 \mathrm{~h}$ so that it would eat a novel diet when one was made available.

Step 3. Following food deprivation, each observer was offered, for $1 \mathrm{~h}$, a weighed sample of a palatable, nutritionally adequate, casein-and-cornstarch-based diet, referred to below as Diet NPT (Normal Protein Test Diet; Teklad Diets, Madison, WI).

Step 4. Immediately following completion of $1 \mathrm{~h}$ of feeding on Diet NPT, each observer was injected intraperitoneally (i.p.) with $0.75 \%$ of body weight of solution: the observers in each of three $\mathrm{LiCl}$ groups were injected with $1 \% \mathrm{w} / \mathrm{vol} \mathrm{LiCl}$ solution; the observers in a saline group (Sal group) were injected with an equal quantity of isotonic saline.

Step 5. One hour following injection, Purina Rodent Lab Chow pellets were placed in each observer's cage, and each observer was given $24 \mathrm{~h}$ to recover from the effects of the injection.

Step 6. Twenty-four hours following injection of the observers, each demonstrator (which had been food-deprived for $23 \mathrm{~h}$ and had been habituated, for 2 days, to a 23-h food-deprivation schedule) was offered a weighed sample of Diet NPT for $1 \mathrm{~h}$ in its home cage.

Step 7. Immediately after each demonstrator had been fed Diet NPT, it was injected i.p. with $2 \%$ of body weight of either $1 \% \mathrm{w} / \mathrm{vol} \mathrm{LiCl}$ solution or an equal quantity of isotonic saline.

Step 8. Immediately following injection, each demonstrator was introduced into the cage of an observer and allowed to interact with that observer for $30 \mathrm{~min}$. Twelve observers (2-poison-dems, $\mathrm{LiCl}$ group) each interacted with 2 demonstrators in succession, both of which had been injected with $\mathrm{LiCl}$, and 12 observers (2-dems, Sal group) each interacted with 2 demonstrators in succession, both of which had been injected with isotonic saline. A third group of 12 observers (2-cups, $\mathrm{LiCl}$ group) was presented for $30 \mathrm{~min}$ with two food cups in succession, each containing a weighed sample of Diet NPT. A fourth group of 12 observers (Sal group) interacted with nothing during Step 8.

Step 9. At the end of the 1-h period of interaction, each observer was offered a choice, for $22 \mathrm{~h}$, between two weighed food cups, one containing Diet NPT and one containing Diet Coc, an unfamiliar diet composed of powdered Purina Rodent Lab Chow adulterated $2 \%$ by weight with Hershey's cocoa.
At the end of the 22-h test period, the experimenter weighed both food cups and determined the percentage of Diet NPT eaten by each observer.

Because the experiment required that (1) observers develop an aversion to Diet NPT, (2) demonstrators eat Diet NPT, and (3) observers choose between diets during testing, we discarded data from (1) observers ( $n=3$ ) that failed to eat $2 \mathrm{~g}$ of Diet NPT during Step 3, (2) observers $(n=1)$ whose demonstrators failed to eat $2 \mathrm{~g}$ of Diet NPT during Step 6 , and (3) observers $(n=1)$ that failed to eat a total of $5 \mathrm{~g}$ of food during Step 9 .

\section{Results and Discussion}

The main results of Experiment 1 are presented in Figure 2, which shows the mean amount of Diet NPT ingested by the observers in the four groups as a percentage of the total amount the observers ate during testing (Step 9 of procedure). As can be seen in Figure 2, there was a significant effect of treatment on intake of Diet NPT: the observers in the 2-cups, $\mathrm{LiCl}$ group ate significantly less Diet NPT than did the observers in the other three groups. The relatively small intake of Diet NPT by the observers in the 2-cups, $\mathrm{LiCl}$ group in comparison with the Sal group indicates that we were successful in inducing an aversion to Diet NPT in the three groups of observers injected with $\mathrm{LiCl}$.

The finding that a previously acquired aversion to Diet NPT can be reversed by exposure to 2 demonstrators fed Diet NPT, but not by exposure to two food cups containing Diet NPT, replicates an earlier finding in our laboratory (Galef, 1986b). The finding that exposure to 2 poisoned demonstrators fed Diet NPT was as effective in reversing a learned aversion to Diet NPT as was exposure to 2 unpoisoned demonstrators fed Diet NPT is consistent with the results of previous experiments conducted both in our laboratory (Galef et al., 1983) and elsewhere (A. Posadas-Andrews, personal communication, 1982), indicating that observer rats develop preferences for, not aversions to, diets eaten by ill demonstrators with which they interact.

It might be argued that the observers in the present experiment responded similarly to interaction with $\mathrm{LiCl}-$ and saline-injected demonstrators because they could not dis- 


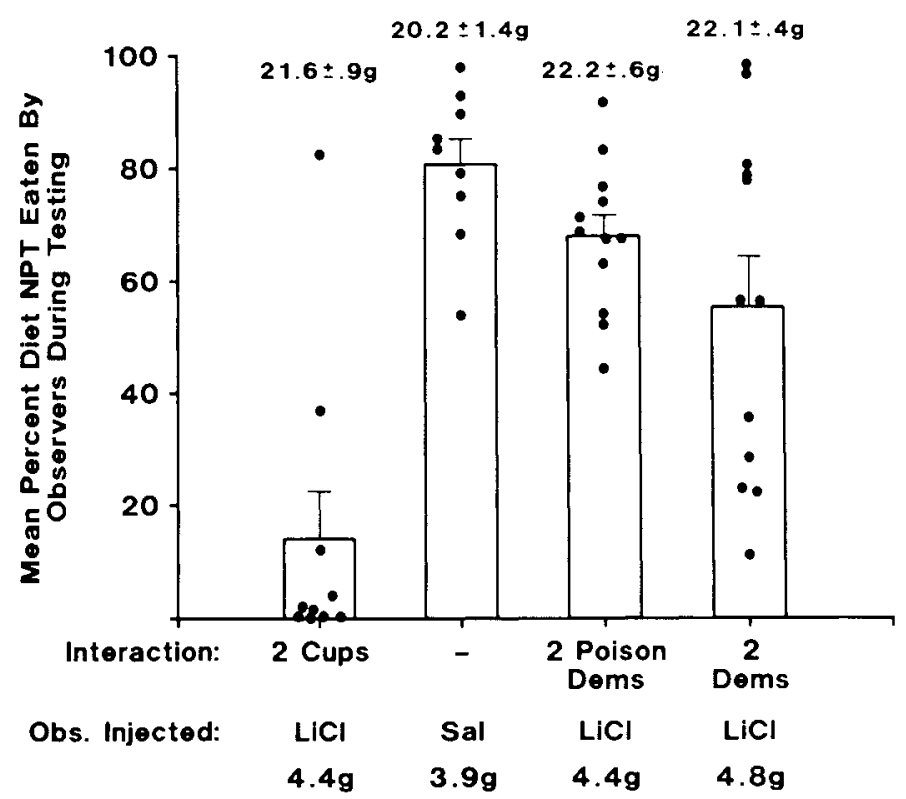

Figure 2. Mean amount of Diet NPT eaten by observers in Experiment 1 as a percentage of the total amount they ingested during testing. Bars $= \pm 1$ $S E M$, points are individual data points, numbers above histograms = mean total grams $( \pm 1 S E M)$ eaten by observers during testing, numbers below abscissa $=$ mean grams eaten by observers during Step 3 of the procedure.

criminate between them. However, the dose of $\mathrm{LiCl}$ with which we injected the demonstrators was the same as that used by Lavin, Freise, and Coombes (1980) in demonstrations of the "poison-partner effect," which requires different responses by observers to $\mathrm{LiCl}$ - and salineinjected rats. Furthermore, all $\mathrm{LiCl}$-injected demonstrators, but no saline-injected demonstrators, had clearly observable diarrhea while interacting with their respective observers.

\section{EXPERIMENT 2}

In Experiment 1, we found that interaction with ill demonstrators that had eaten Diet NPT was as effective in reversing a previously acquired aversion to Diet NPT as was interaction with healthy demonstrators that had eaten Diet NPT. In Experiment 2, we investigated the effects of preexposure of observer rats to healthy and ill demonstrators fed Diet NPT on subsequent learning by those observer rats of a toxicosis-induced aversion to Diet NPT.

\section{Method}

\section{Subjects}

Fifty experimentally naive 42-day-old female Long-Evans rats from the McMaster vivarium served as observers in the present experiment. It was our intention to assign 10 subjects randomly to each of the five conditions described in Step 2 of the procedure. However, an error in our subject-assignment algorithm led to observer group sizes ranging from 8 to 14 .

An additional 50 49- to 56-day-old female rats from the same source served as demonstrators. Many of these demonstrators had served as observers in previous experiments.

\section{Apparatus and Foods}

The apparatus and foods used in the present experiment were the same as those used in Experiment 1.

\section{Procedure}

Experiment 2 was conducted in eight Steps, described below (see Figure 3 for schematic of treatment of subjects).

Step 1 . Both the observers and the demonstrators were placed on a 23-h food-deprivation schedule, eating powdered Purina Rodent Lab Chow for $1 \mathrm{~h}$ /day for 2 consecutive days.

Step 2. Following a third 23-h period of food deprivation of both demonstrators and observers, 36 demonstrators were each offered, for $1 \mathrm{~h}$, a weighed food cup containing Diet NPT.

Step 3. Immediately after feeding, each demonstrator was injected i.p. with either $2 \%$ of body weight, $1 \%$ w/vol $\mathrm{LiCl}$ solution ( $n=$ $20)$ or an equal quantity of isotonic saline solution $(n=16)$.

Step 4. Immediately after injection, each of 18 demonstrators was placed in the home cage of an observer and allowed to interact with the observer for $15 \mathrm{~min}$. At the end of this 15 -min period, members of this first squad of demonstrators were removed from the cages of their respective observers and replaced with a second squad of 18 demonstrators, each of which had just completed a 1-h period of eating Diet NPT and had then been injected with either $\mathrm{LiCl}$ or isotonic saline solution. Eight observers (2-dems-fed-NPT, LiCl group) interacted with 2 demonstrators in succession, each fed Diet NPT and injected with saline solution, and 10 observers (2-poison-dems-fed-NPT, LiCl group) interacted with 2 demonstrators in succession, each fed Diet NPT and made ill by injection with $\mathrm{LiCl}$ solution.

An additional 8 observers (2-dems-not-fed, $\mathrm{LiCl}$ group) each interacted for $30 \mathrm{~min}$ with 2 demonstrators in succession (15 min each) that had not been fed before they were placed in the cages of their respective observers, but that had been injected with $1 \% \mathrm{w} / \mathrm{vol} \mathrm{LiCl}$.

Twenty-four observers assigned to two control groups did not interact with any demonstrators during Step 4 of the experiment.

Step 5. All 50 observers were offered, for $15 \mathrm{~min}$, weighed food bowls containing Diet NPT. Two observers failed to eat any Diet NPT and were discarded from the experiment. 


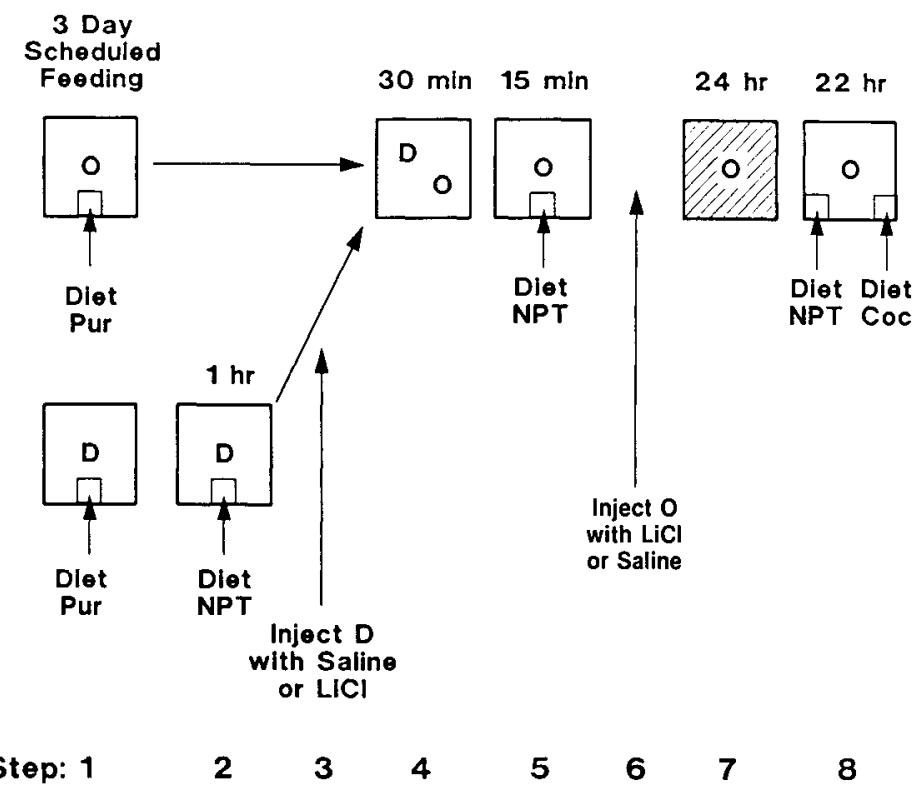

Figure 3. Schematic of the procedure of Experiment 2. D = demonstrator, $\mathbf{O}=$ observer, hatching $=$ ad-lib access to Purina Rodent Lab Chow pellets.

Step 6. Immediately after eating Diet NPT for $15 \mathrm{~min}$, each observer was injected with $0.75 \%$ of body weight of solution, either $1 \% \mathrm{w} / \mathrm{vol} \mathrm{LiCl}$ solution $(n=39)$ or an equal quantity of isotonic saline $(n=9)$.

Step 7. All observers were given $24 \mathrm{~h}$ to recover from the effects of injection. During this $24-\mathrm{h}$ period, they had ad-lib access to Purina Rodent Lab Chow pellets.

Step 8 . Following recovery, each observer was offered, for $22 \mathrm{~h}$, a choice between Diet NPT and Diet Coc, an unfamiliar food.

\section{Results and Discussion}

The main results of Experiment 2 are presented in Figure 4, which shows the mean amount of Diet NPT eaten by the observers during testing (Step 8) as a function of their treatment during Steps 4 and 6 of the procedure.

As can be seen by examination of the two histograms on the left side of Figure 4, the observers in the control

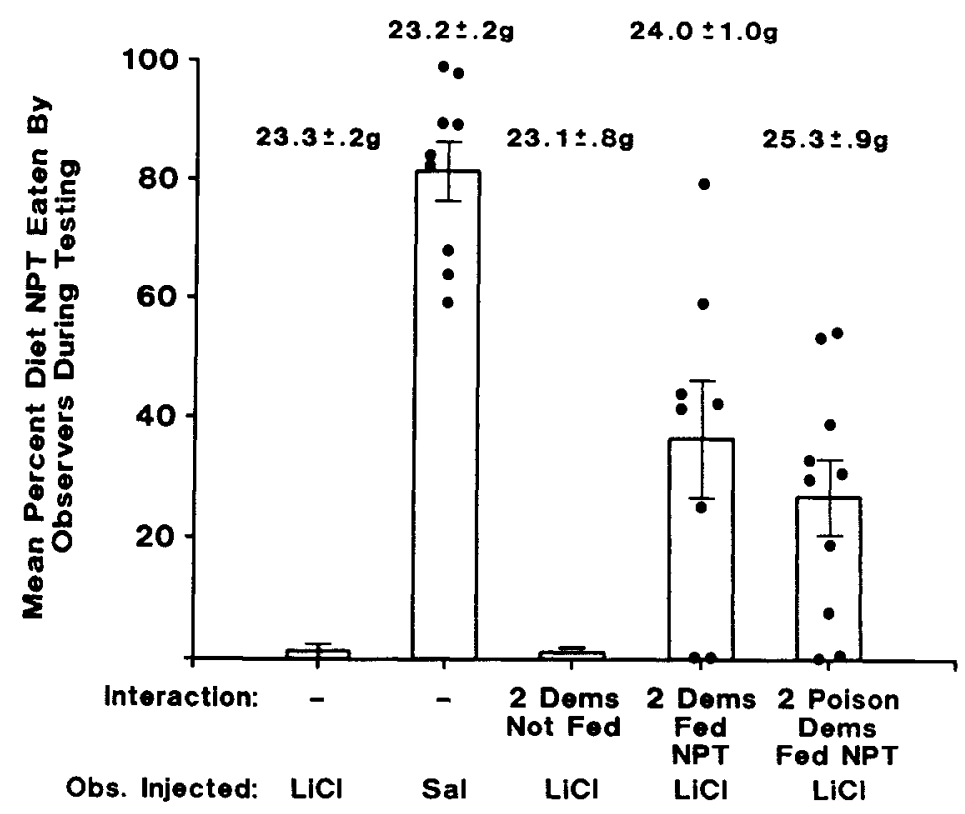

Figure 4. Mean amount of Diet NPT eaten by observers in Experiment 2 as a percentage of the total amount they ingested during testing. Bars $= \pm 1 S E M$, points are individual data points, numbers above histograms = mean total grams ( \pm 1 SEM) eaten by observers during testing. 
group (i.e., the observers that did not interact with demonstrators during Step 4) that were injected with $\mathrm{LiCl}$ during Step 6 ate far less Diet NPT during testing (Step 8) than did the observers in the control group that were injected with saline solution during Step 6. Our attempt to induce an aversion in the observers was successful.

The more interesting data is to be found in the three histograms on the right side of Figure 4, which describe the behavior during testing (Step 8) of the observers that had interacted with demonstrators during Step 4 and were injected with $\mathrm{LiCl}$ during Step 6 . The observers that interacted with unfed demonstrators during Step 4 and were poisoned during Step 6 exhibited aversions to Diet NPT during testing that was indistinguishable from the aversions exhibited by observers that did not interact with demonstrators during Step 4 and were poisoned in Step 6. As might be expected, simply interacting with conspecifics before eating an unfamiliar diet and becoming ill did not interfere with taste-toxicosis conditioning.

On the other hand, interaction with demonstrators fed Diet NPT, whether ill or healthy, did attenuate subsequent learning of an aversion to Diet NPT. The observers that interacted with poisoned demonstrators fed Diet NPT during Step 4 and the observers that interacted with healthy demonstrators fed Diet NPT during Step 4 both ate a significantly greater percentage of Diet NPT during testing than did the observers that interacted with unfed demonstrators during Step $4[F(1,2)=7.59, p<.004$; Newman-Keuls test, both $p s<.01]$. Previous interaction with poisoned and unpoisoned demonstrators fed Diet NPT had equivalent effects on subsequent learning of an aversion to Diet NPT by observers (Newman-Keuls test, n.s.). Once again, observers did not respond differently to interaction with poisoned and unpoisoned demonstrators.

\section{EXPERIMENT 3}

In Experiment 3, observers interacted with demonstrators fed either a cinnamon- or a cocoa-flavored diet, ate both cinnamon- and cocoa-flavored diets in succession, and were then poisoned. Later, they were given a choice test between cinnamon- and cocoa-flavored diets (Galef et al., 1983). In this paradigm, the diet choices of observers reflect the effects of interaction with demonstrators on observers' later probabilities of learning aversions to each of the two diets that they had eaten before becoming ill.

\section{Method}

Subjects

Thirty-two experimentally naive 42-day-old male Long-Evans rats from the McMaster vivarium served as observers. An additional 32 49- to 56-day-old males from the same source served as demonstrators.

\section{Apparatus and Foods}

The apparatus was the same as that used in Experiment 1. Two foods were used in the present experiment: a cinnamon-flavored diet (Diet Cin; powdered Purina Rodent Lab Chow adulterated 1\% by weight with McCormick's pure ground cinnamon) and a cocoaflavored diet (Diet Coc; powdered Purina Rodent Lab Chow adulterated $2 \%$ by weight with Hershey's cocoa).

\section{Procedure}

The present experiment was conducted in nine steps (see Figure 5).

Step 1 . Both the observers and the demonstrators were placed on a 23-h food-deprivation schedule, eating powdered Purina Rodent Lab Chow for $1 \mathrm{~h} /$ day for 2 consecutive days.

Step 2. Following a third 23-h period of food deprivation of both demonstrators and observers, each demonstrator was fed either Diet Cin or Diet Coc for $1 \mathrm{~h}$.

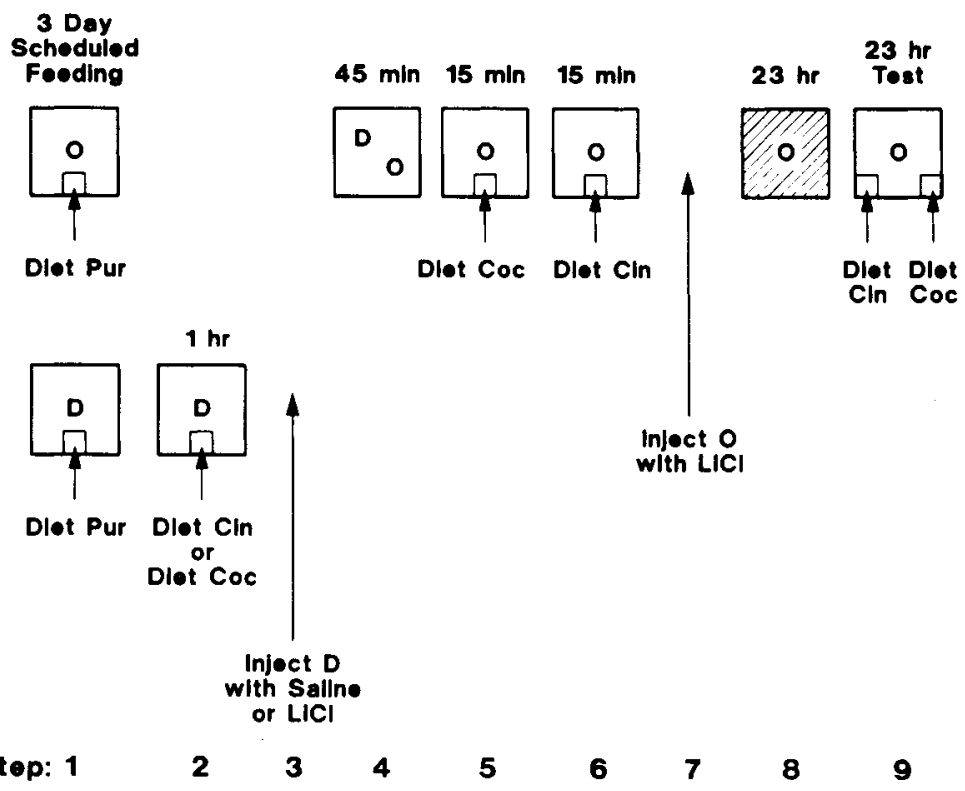

Figure 5. Schematic of the procedure of Experiment 3. $D=$ demonstrator, $O=$ observer, hatching $=$ ad-lib access to Purina Rodent Lab Chow pellets. 
Step 3. Each demonstrator was then injected with $1 \%$ of body weight of either isotonic saline solution or $2 \% \mathrm{w} / \mathrm{vol} \mathrm{LiCl}$ solution.

Step 4. Immediately after injection, each demonstrator was placed in the cage of an observer. The demonstrator and the observer were allowed to interact for $45 \mathrm{~min}$.

Step 5. At the end of the 45-min period of interaction, the demonstrator was removed from each observer's cage and replaced with a weighed food cup containing Diet Coc. This food cup was left in the observer's cage for $15 \mathrm{~min}$.

Step 6. At the end of this first 15-min feeding period, the first food cup was removed from each observer's cage and replaced, for $15 \mathrm{~min}$, with a second food cup containing Diet Cin.

Step 7. Immediately following termination of the second feeding period, each observer was injected i.p. with $2 \%$ of body weight of $1 \% \mathrm{w} / \mathrm{vol} \mathrm{LiCl}$ solution.

Step 8. One hour following injection, Purina Rodent Lab Chow pellets were placed in cach observer's cage, and each observer was given $23 \mathrm{~h}$ to recover from the effects of toxicosis.

Step 9 . Following the 23 -h recovery period, each observer was offered, for $23 \mathrm{~h}$, a simultaneous choice of weighed samples of Diet $C$ in and Diet Coc. At the end of the 23-h test period, the experimenter determined each observer's intake of Diet Cin and Diet Coc and calculated the percentage of Diet Coc eaten by each observer.

\section{Results and Discussion}

The main results of Experiment 3 are presented in Figure 6, which shows the mean amount of Diet Coc eaten by the observers during testing as a percentage of their total intake during the test period (Step 9 of the procedure). As is clear from examination of Figure 6, the observers tended to form aversions to whichever diet their respective demonstrators had not eaten, and there was no effect of the solution with which demonstrators were injected during Step 3 of the procedure on their respective observers' diet choices during Step 9. Once again, the observers seem to have responded to the foods eaten by their respective demonstrators, but not to the state of their respective demonstrators' health.

We repeated Experiment 3, but varied some parameters: demonstrators and their observers interacted for $1 \mathrm{~h}$ during Step 4 (rather than for $45 \mathrm{~min}$ ), demonstrators were injected with $1 \%$ rather than $2 \% \mathrm{w} / \mathrm{vol} \mathrm{LiCl}$ solution during Step 3, and individual demonstrators and observers were maintained together in pairs rather than in isolation during Step 1. The outcome of the replication was identical to that of the main experiment. Once again, during testing, observers ate the diet that their respective demonstrators had eaten during Step 2 and were not affected by the substance with which their respective demonstrators were injected during Step 3. We again failed to find evidence of observers' response to the state of health of their demonstrators.

\section{GENERAL DISCUSSION}

When one describes studies of demonstrator-induced enhancement of the diet preferences of naive observer rats to an audience of experimental psychologists or biologists, someone in the audience is sure to ask whether interaction of a naive observer rat with a sick demonstrator leads the observer to avoid the food eaten by the sick demonstrator. Intuitively, it seems a better strategy for rats to avoid unfamiliar food that ill conspecifics have eaten than to ingest any food that any conspecific, whether healthy or ill, has eaten. From an adaptationist perspective, it is something of a puzzle that no evidence of response to the

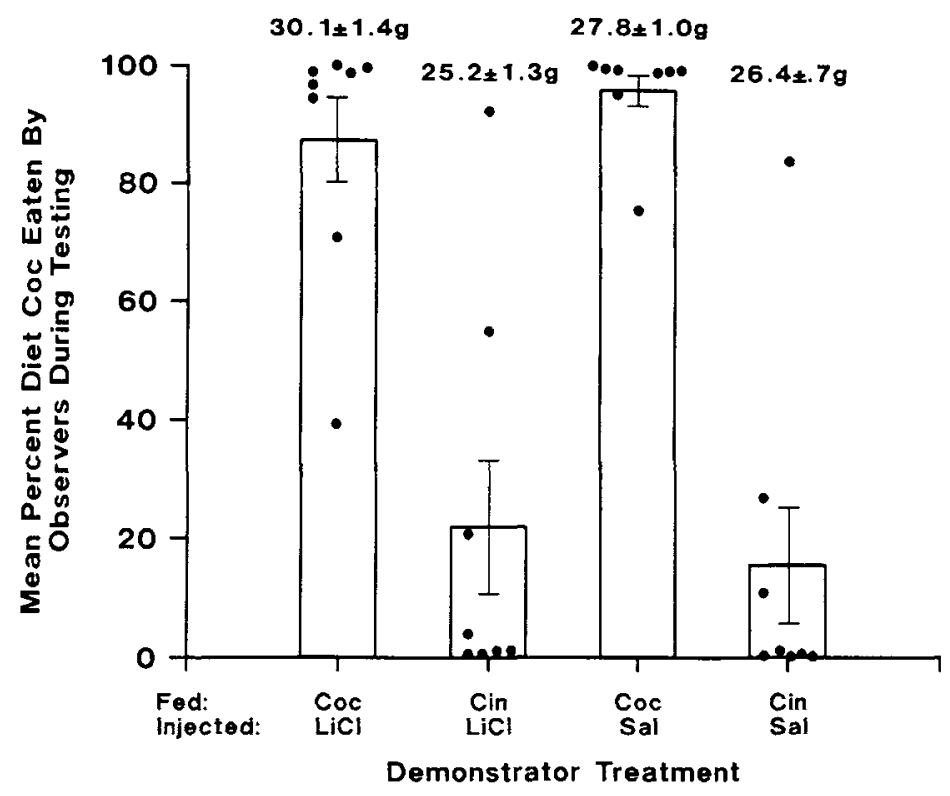

Figure 6. Mean amount of Diet Coc eaten by observers in Experiment 3 as a percentage of the total amount they ingested during testing. Bars $= \pm 1 S E M$, points are individual data points, numbers above histograms = mean total grams ( $\pm 1 S E M$ ) eaten by observers during testing. 
well-being of demonstrators has been found in their observers. Rats can respond differently to healthy and ill conspecifics (Bond, 1982; Coombes, Revusky, \& Lett, 1980; Galef et al., 1983; Gemberling, 1984; Lavin et al., 1980); they simply do not seem to use this ability in deciding whether to eat foods those conspecifics have consumed. The nature of the olfactory messages passing from demonstrators to observers concerning the foods demonstrators have eaten (Galef, Mason, Preti, \& Bean, 1988; Galef \& Stein, 1985) seems to preclude communications concerning avoidance of toxic foods. Data from a dozen experiments are all consistent with the same conclusion: Rats can learn from others which foods to eat, but not which foods to avoid eating (Galef, 1985a).

\section{REFERENCES}

BoND, N. W. (1982). Transferred odor aversions in adult rats. Behavioral \& Neural Biology, 35, 417-421.

Coombes, S., Revusky, S. H., \& LeTt, B. T. (1980). Long-delay tasteaversion learning in an unpoisoned rat: Exposure to a poisoned rat as the unconditioned stimulus. Learning \& Motivation, 11, 256-266.

GALEF, B. G., JR. (1985a). Direct and indirect behavioral pathways to the social transmission of food avoidance. In P. Bronstein \& N. S. Braveman (Eds.), Experimental assessments and clinical applications of conditioned food aversions (pp. 203-215). New York: New York Academy of Sciences.

GALEF, B. G., JR. (1985b). Socially induced diet preference can partially reverse a $\mathrm{LiCl}$-induced diet aversion. Animal Learning \& Behavior, 13, 415-418.

GALEF, B. G., JR. (1986a). Social identification of toxic diets by Norway rats. Journal of Comparative Psychology, 100, 331-334.

GALEF, B. G., JR. (1986b). Social interaction modifies learned aversions, sodium appetite, and both palatability and handling-time induced dietary preference in rats (Rattus norvegicus). Journal of Comparative Psychology, 100, 432-439.

GALEF, B. G., JR. (1987). Social influences on the identification of toxic foods by Norway rats. Animal Learning \& Behavior, 15, 327-332.
GALEF, B. G., JR. (1989). Socially mediated attenuation of taste-aversion learning in Norway rats: Preventing development of "food phobias." Animal Learning \& Behavior, 17, 468-474.

Galef, B. G., Jr., Mason, J. R., Preti, G., \& Bean, N. J. (1988). Carbon disulfide: A semiochemical mediating socially-induced diet choice in rats. Physiology \& Behavior, 42, 119-124.

GALEF, B. G., JR., \& StEIN, M. (1985). Demonstrator influence on observer diet preference: Analyses of critical social interactions and olfactory signals. Animal Learning \& Behavior, 13, 31-38.

GALEF, B. G., JR., WIGMORE, S. W. (1983). Transfer of information concerning distant foods: A laboratory investigation of the 'information-centre' hypothesis. Animal Behaviour, 31, 748-758.

GAlef, B. G., Jr., Wigmore, S. W., Kennett, D. J. (1983). A failure to find socially mediated taste aversion learning in Norway rats ( $R$. norvegicus). Joumal of Comparative Psychology, $97,358-363$.

Gemberling, G. (1984). Ingestion of a novel flavor before exposure to pups injected with lithium chloride produces a taste aversion in mother rats. Joumal of Comparative Psychology, 98, 285-301.

Grover, C. A., Kixmiller, J. S., Erickson, C. A., Becker, A. H., DAvis, S. F., NAllaN, G. B. (1988). The social transmission of information concerning aversively conditioned liquids. Psychological Record, 38, 557-566.

Heyes, C. M., \& Durlach, P. J. (1990). "Social blockade" of taste aversion learning in Norway rats. ( $R$. norvegicus): Is it a social phenomenon? Journal of Comparative Psychology, 104, 82-87.

LAvin, M. J., Freise, B., CoOMBes, S. (1980). Transferred flavor aversions in adult rats. Behavioral \& Neural Biology, 28, 15-33.

Posadas-Andrews, A., \& Roper, T. J. (1983). Social transmission of food preferences in adult rats. Animal Behaviour, 31, 265-271.

Richard, M. M., Grover, C. A., \& Davis, S. F. (1987). Galef's transfer of information effect occurs in a free-foraging situation. Psychological Record, 37, 79-87.

Valsecchi, P., Galef, B. G., JR. (in press). Social influences on the food preferences of house mice (Mus musculus): A comparative analysis of behavioral processes. International Joumal of Comparative Psychology.

(Manuscript received May 17, 1989; revision accepted for publication October $24,1989$. 